\title{
Heterozygous HTRA1 missense mutation in CADASIL-like family disease
}

\author{
Xiaowei Wu, Changxin Li, Jinming Mao, Ling Li, Yan Liu and Yao Hou \\ Department of Neurology, the First Hospital of Shanxi Medical University, Taiyuan, China
}

\begin{abstract}
The aim of this study was to find related pathogenic genes in cerebral autosomal dominant arteriopathy with subcortical infarcts and leukoencephalopathy in (CADASIL)-like patients. The direct sequencing and high-throughput multiplex polymerase chain reaction (PCR) was performed to screen for related genes. The clinical and imaging data of a CADASIL-like patient (the proband) and his family members were collected. At first, the known hereditary cerebral vascular genes of the pro-band were screened with direct sequencing to find candidate gene mutations. High-throughput multiplex PCR was then used to analyze the single nucleotide polymorphism of the candidate gene in the family members. The results showed that there was missense mutation of the high temperature requirement protease A1 (HTRA1) gene in the pro-band, which may be a pathogenic factor according to the biological software analysis. The following SNP results revealed that the other family members also had the HTRA1 gene mutation. Thus, the CADASIL-like family disease may be caused by heterozygous HTRA1 gene mutation, which leads to autosomal dominant hereditary cerebral small vessel disease.
\end{abstract}

Key words: HTRA1; CADASIL-like; SNP; Cerebral small vessel disease

\section{Introduction}

Cerebral small vessel disease (CSVD) is a set of heterogeneous diseases due to the pathological changes of cerebral arterioles, venules and capillaries (1-3). Stroke and cognitive impairment are the main clinical features of CSVD, which is mainly characterized by cerebral white matter lesions such as lacunar infarction and microhemorrhage in clinical imaging.

CSVD includes the sporadic and the familial hereditary cerebral small vessel disease. Sporadic CSVD is related to aging and hypertension, while the familial hereditary CSVD frequently starts early and can be inherited in an autosomal dominant inheritance manner (3-5). It has been confirmed that familial hereditary CSVD is related to certain types of mutation of a single gene. Cerebral autosomal dominant with subcortical infarcts and leukoencephalopathy (CADASIL) was associated with Notch3 mutation (6). Hereditary cerebral amyloid angiopathy is caused by COL4A1/COL4A2 mutation $(7,8)$, and CARASIL by the high temperature requirement protease A1 HTRA1 allele mutations, which is also called HTRA1 homozygous mutations (9-13).

However, only very few patients have a clear gene mutation and the cause of the majority of familial cerebral small vessel disease cases is still unknown. To find out the probable gene mutation location, we analyzed a patient who was diagnosed as Notch3-negative but CADASIL-like (14).
The hereditary cerebral vascular gene screening by direct sequencing and the single nucleotide polymorphism (SNP) of the candidate gene were analyzed by high-throughput multiplex polymerase chain reaction to find the causative gene of the family cerebrovascular disease.

\section{Material and Methods}

\section{Patient}

Clinical data was collected from a case of a suspected CADASIL patient and his family members in the Department of Neurology at the first hospital of Shanxi Medical University. The Notch 3 gene consists of 33 exons, and we have examined 29 exons except the 4 exons 17/21/22/23, which do not belong to the hot spot mutations. The genetic diagnosis of Notch3 and skin biopsy of the patient were both negative. The patient and his family members who participated in the study all gave written informed consent.

According to our investigation, the pro-band did not have alopecia, spondylosis, and adult-onset Alexander's disease. Pedigree analysis was performed for the proband and his family, and there were two major features: 1) stroke and progressive cognitive impairment as the major clinical characteristics; 2) negative Notch3. Stroke is an ischemic or infarct injury of the brain that results in

Correspondence: Changxin Li: <changxin_li123@163.com>

Received July 25, 2017| Accepted November 6, 2017 
neurological deficit and contralateral impairments in verbal, cognition, motor and sensory system. Stroke and cognitive impairment are inter-related.

\section{Blood samples}

Blood samples of the pro-band were collected and used for screening the hereditary cerebrovascular disease related genes, such as HTRA1, APP, CST3, and ITM2B, etc., and SNP genotyping assay were then conducted on family members after identifying the candidate gene.

\section{Genetic sequencing}

The genetic sequencing of the 27 related genes of hereditary cerebrovascular disease were conducted. The 27 genes included NOTCH3, HTRA1, KRIT1 (CCM1), CCM2, PDCD10 (CCM3), GLA, HBB, CBS, MMADHC, MTHFR, MTR, MTRR, PRKAR1A, F5, APP, CST3, ITM2B, ENG, ACVRL1, SMAD4, ABCD1, ARSA PLP1, MLC1, $A S P A, G F A P$, and $G A L C$.

\section{SNP genotyping assay for the candidate gene}

To extract DNA, EDTA was added to venous blood $(2 \mathrm{~mL}$ ) for anticoagulation, and the column method (QIAamp Blood DNA Mini Kit, Qiagen,USA) was used for extracting genomic DNA from peripheral blood leukocytes. DNA was stored at $4^{\circ} \mathrm{C}$.

Gene sequences were obtained from the human genome database GenBank. The Primer Premier 5.0 software was applied to design primers of the HTRA1 gene exon coding region, using 2 X PCR MasterMix polymerase (Tiangen) for PCR amplification (ABI9700 PCR Instruments, Life Technology, USA). The PCR products from the specific primers were direct sequenced by Taiyuan Jinyu Clinical Inspection Ltd. using ABI3500 (Life Technology, USA). Then, reference sequences (NM_002775.4 and NG_011554.1) were used for comparison to localize the possible gene mutation.

\section{Bioinformatics analysis}

Sequencing results were analyzed by PolyPhen2 (Polymorphism Phenotyping v2, a tool that can predict the possible impact of an amino acid substitution on the structure and function of protein), SIFT (sorting intolerant from tolerant) and Mutation Taster to see whether there is a likelihood of pathogenesis by gene mutation. SIFT predicts whether an amino acid substitution can affect protein function and prediction is based on the degree of conservation of amino acid residues in sequence alignments derived from closely related sequences, collected through PSI-BLAST.

\section{Results}

Clinical data of the patient and his family members Based on the pedigree chart of the pro-band (Figure 1), there was a clear family history of cerebrovascular disease on the paternal side, while the disease did not exist on the maternal side, inferring that the disease was originated from paternal autosomal dominant cerebrovascular disease. In the 17 people of the three generations, there were 6 with early-onset of cerebral vascular disease except the pro-band. Among the 6 people, there were 4 who had been considered genetically correlated for cerebral vascular disease, $3(11,114,1117)$ were already deceased, and one (III6) was alive. Moreover, one person was considered "patient", based on imaging diagnosis. II5, III1, and III4 had symptoms of cerebral vascular disease, but lacked imaging data, therefore they were not considered genetically related for the disease. The cause of death of II2 was unknown (might have been caused by cerebrovascular disease).

The pro-band, with onset of the disease at the age of 35 years, had no hypertension, diabetes, smoking or other risk factors of cerebrovascular disease, and there was no family history of cerebrovascular disease. In 2006, he presented with dizziness and double lower extremity weakness. In 2008, 2009, and 2011, he presented with similar symptoms, and double lower extremity weakness appeared alternately. After cerebral infarction treatment, the symptoms were slightly reduced. In 2012, he presented with dizziness and double lower limb weakness that were more serious, and required hospitalization. There was no definite risk factor for cerebrovascular disease. On physical examination, findings were clear mind, slower verbal reaction, slightly dull, the tongue to the right, limb muscle strength of grade 5 , hypermyotonia, hyperreflexia, tendon hyperreflexia, bilateral symmetry for sense of depth, and bilateral Babinski syndrome $(+)$. In cranial MRI, findings were multiple lacunar infarction in bilateral basal ganglia, lateral ventricles, and semi-oval center, and changes of bilateral periventricular white matter demyelination.

III6 was the sister of the pro-band, born in May 1955. In 2003, she was diagnosed with cerebral infarction for the first time, and it recurred once a year on average. In 2012, she presented with recurrent right limb numbness and weakness, and slurred speech. Symptoms aggravated in 4 days, and she required hospitalization. On physical examination, she was found to be clear-minded, with slurred speech, shallow right nasolabial fold, tongue to the right, positive right limb paresis test, and right Babinski syndrome $(+)$. The imaging findings were similar to the pro-band.

Head MRI imaging data (Figures 2-4) suggested multiple lacunar infarction and diffuse cerebral white matter lesions. However, no obvious abnormality was observed from the cardiovascular-related examination (including 12 lead echocardiography, carotid artery ultrasound, transcranial Doppler, blood routine examination, and blood biochemical analysis). The diagnosis criteria of the CADASIL/ CADASIL-like disease were satisfied by combining clinical symptoms, imaging data, and positive medical history. However, subsequent Notch3 gene test and skin biopsy excluded CADASIL diagnosis. Therefore, the disease was initially classified as CADASIL-like (15), or autosomal 


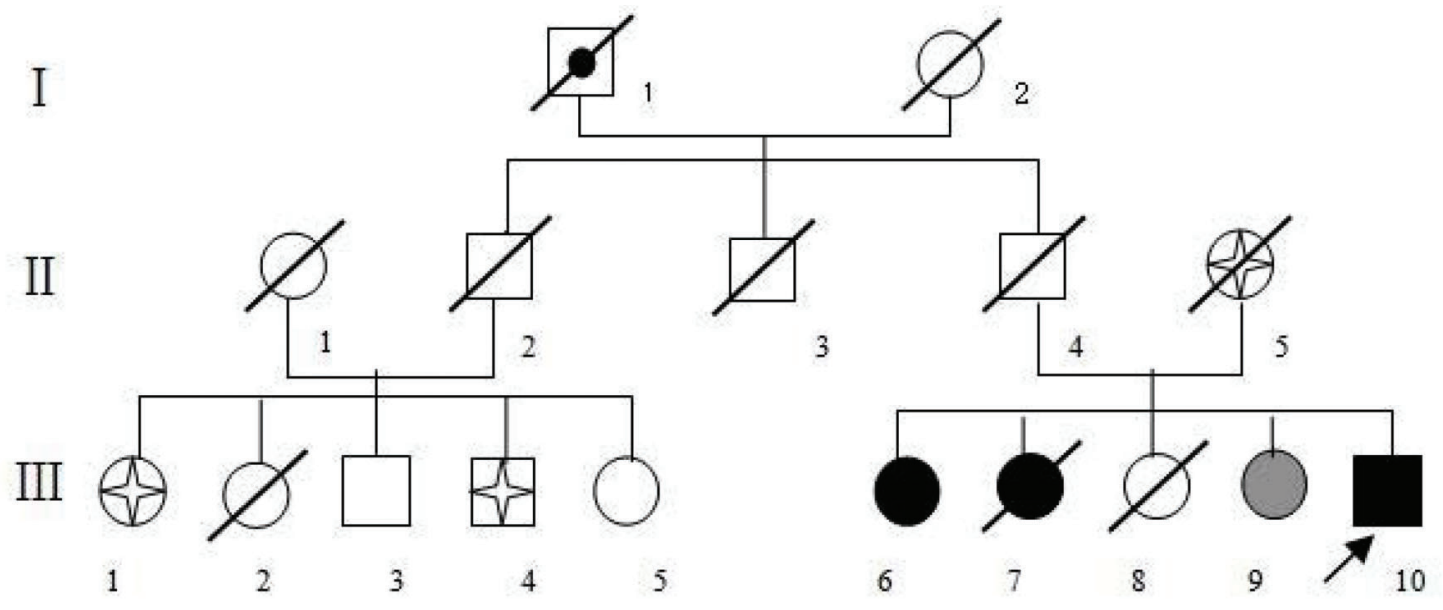

$\square$ heathy male
o heathy female
sick male
sick female
$\nearrow$ pro-band
$/$ deceased

female patients based on clinical or family history (none)

male patients based on clinical or family history

$B$ female patients with non-hereditary cerebrovascular disease

(4) male patients with non-hereditary cerebrovascular disease

$\checkmark$ positive imaging data but without being diagnosed as cerebrovascular disease

Figure 1. Pedigree chart of the pro-band.

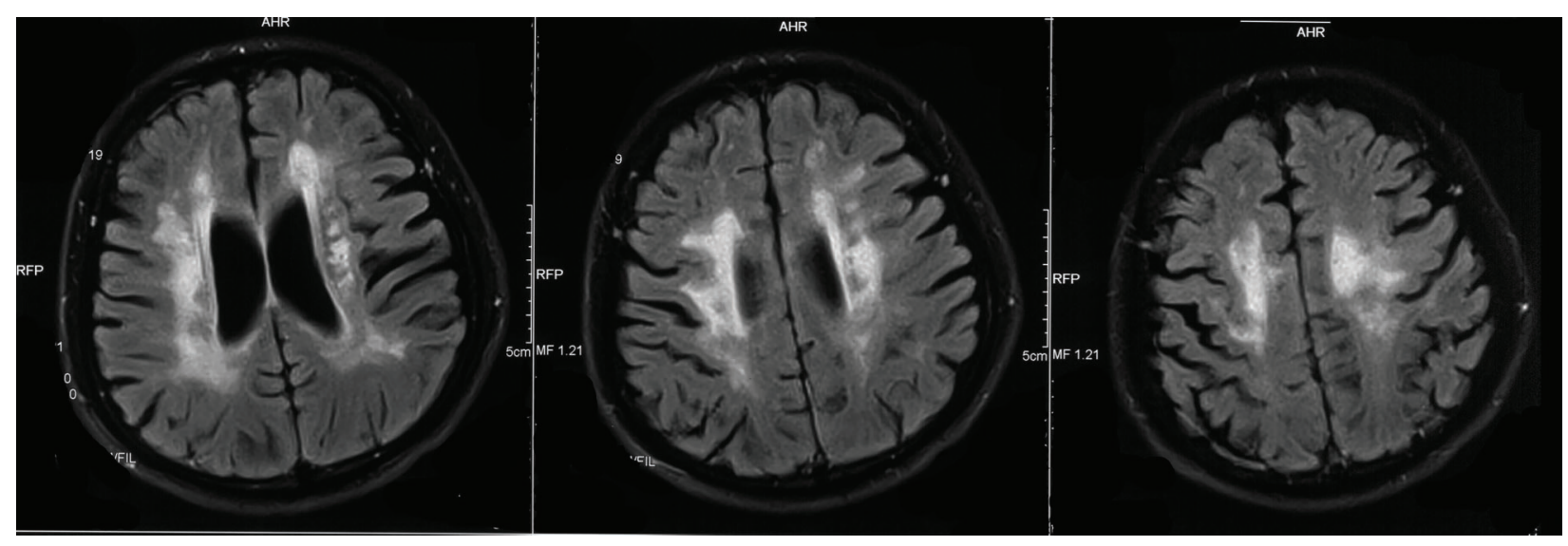

Figure 2. Head FLAIR and MRI images of the pro-band. Image shows bilateral lateral ventricles, bilateral semi-oval center with multiple abnormal signals, and demyelination in the white matter around the bilateral lateral ventricles.

dominant hereditary cerebral small vessel disease without clear genetic diagnosis.

\section{Mini Mental State Examination (MMSE) score}

The pro-band score was 17 on the MMSE, which was low considering that he was a graduate of technical school (normal would be a 24 score), indicating moderate damage of neurologic function. The III6 score was 22, considered low as she had a bachelor degree, indicating slight damage of neurologic function. The III9 score was 28 , and his educational level was bachelor degree, indicating he was normal. 


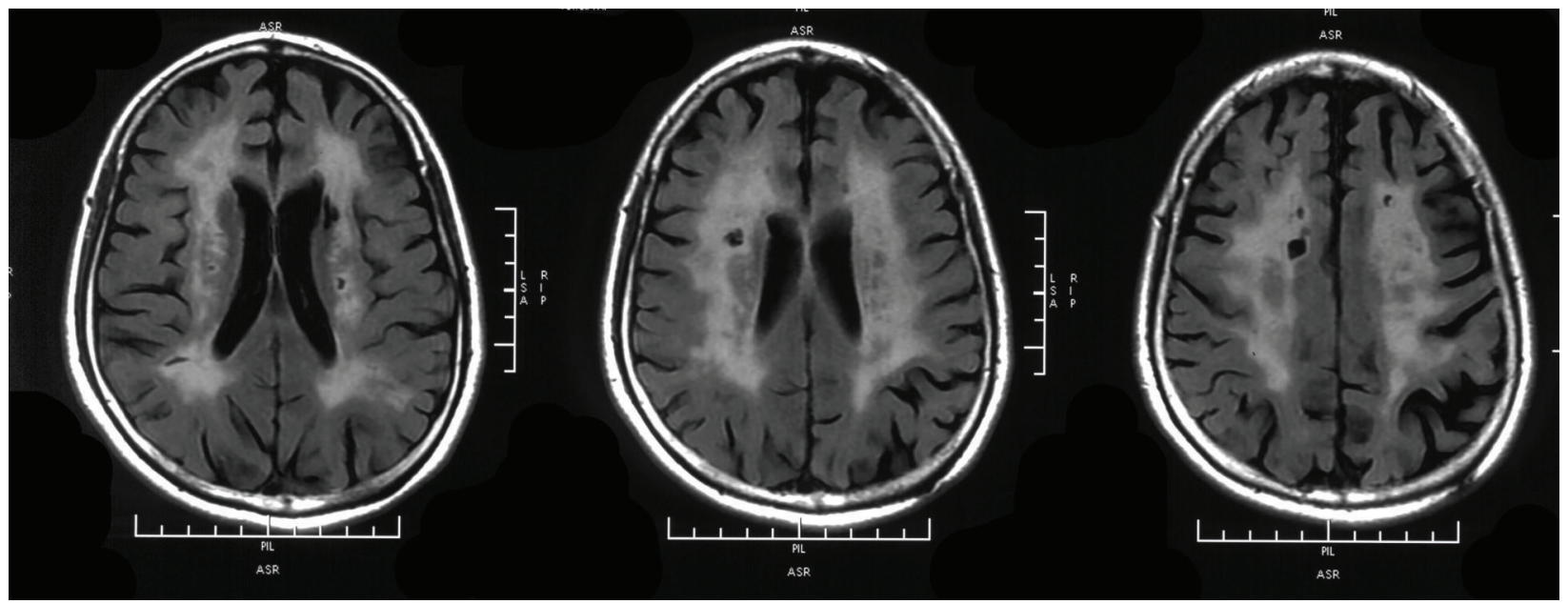

Figure 3. Head MRI and FLAIR images of III6. Image shows bilateral lateral ventricles, bilateral semi-oval center with multiple abnormal signals, and demyelination in the white matter around the bilateral lateral ventricles.

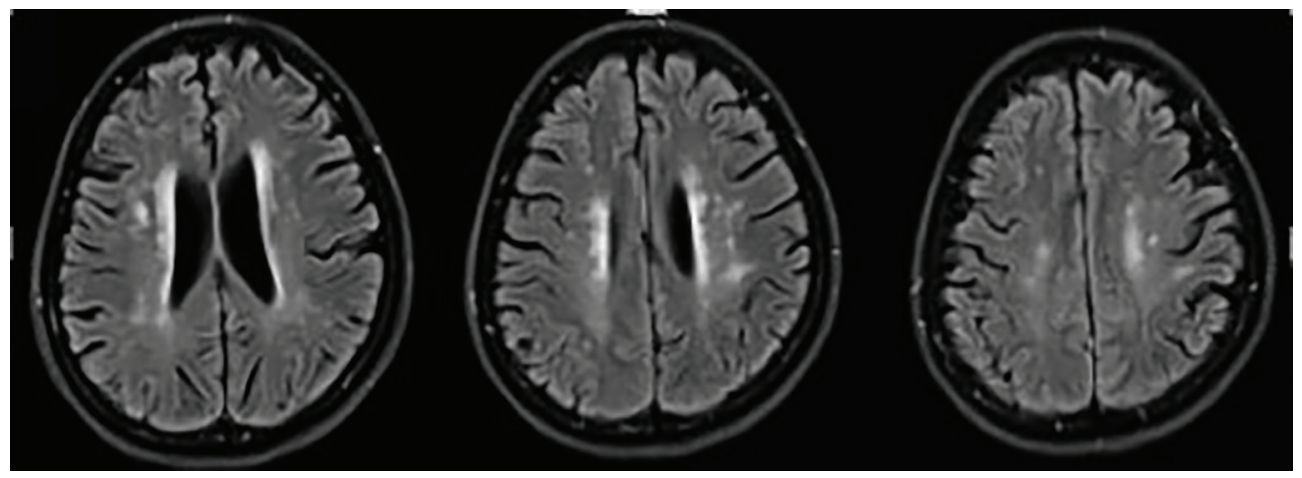

Figure 4. Head MRI and T2WI images of III9 (currently no clinical symptoms). Image shows bilateral lateral ventricles, bilateral semi-oval center with multiple patchy abnormal signal, and the white matter of the bilateral lateral ventricle slightly changed.

\section{Candidate genes and SNP test results}

Direct sequencing was performed for the exons of hereditary cerebrovascular disease related genes from the pro-band, and the sequencing result was then compared with normal gene sequence. No significantly related gene mutations were found. However, a heterozygous missense mutation was detected in HTRA1, i.e., HTRA1 (NM 002775.4) Exon4 c.905G > A p. (Arg302GIn). The biological software analysis with PolyPhen-2, SIFT, and Mutation Taster suggested that this missense mutation very likely caused cerebrovascular disease. Subsequent SNP genotyping assay of the candidate gene in III6 and III9 indicated that the HTRA1 gene had the same single nucleotide polymorphism, strongly suggesting that this heterozygous missense mutation is the reason for family disease (Figure 5).

\section{Discussion}

In this study, a CADASIL-like patient and his family members were investigated. The candidate gene heterozygous
HTRA1 missense mutation was obtained by genetically screening the currently known genes and hereditary cerebrovascular disease related genes. Then, the SNP genotyping assay of the candidate gene was performed for the family members to obtain the preliminary results.

The HTRA 1 gene is located at the long arm of chromosome 10 (10q26) encoding a series of serine protease and its encoding product is widely expressed in vivo (15). This protein family is composed of four domains from $\mathrm{N}$-terminus to $\mathrm{C}$-terminus, including insulin-like growth factor binding domain, Kazal domain, trypsin-like enzyme peptide domain, and PDZ domain. HTRA1 is a gene that encodes a serine protein, which can regulate insulin-like growth factor (IGF) by cleaving the IGR binding protein. In addition, HTRA1 was reported to regulate cell proliferation activity (16).

It is commonly recognized that the CARASIL disease is caused by the mutation of the HTRA1 allele, whereas heterozygous mutations are not pathogenic (17). However, a recent study reported that HTRA1 heterozygous 

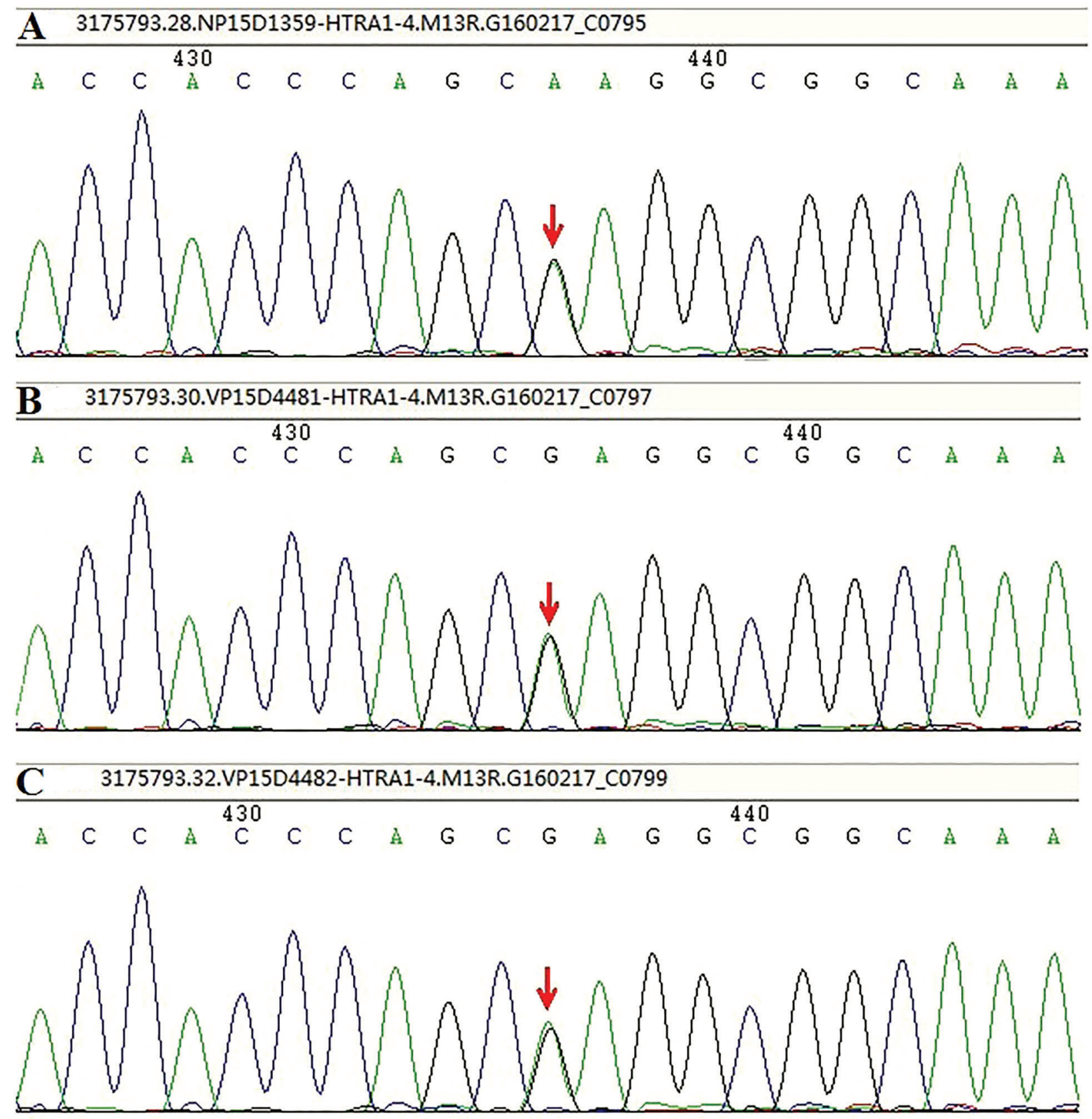

Figure 5. Single nucleotide polymorphism sequencing of candidate genes. Graphs show the $c .905 \mathrm{~g}>\mathrm{A}$ Heterozygous mutation in of the HTRA1 gene of the $(A)$ pro-band (III10), (B) pro-band's eldest sister (III6) and (C) the pro-band's third sister (III9).

mutation could cause autosomal dominant cerebral small vessel disease (18). The conclusion was drawn by selecting candidate genes via gene sequencing, and then the genome comparison was carried out in a relatively large sample of a specific population. The study also indicated that the mutation might account for a large proportion of the non-CADASIL autosomal dominant cerebral small vessel disease cases. The genetic pattern of the disease may be attributed to the change in the content and activity of the encoded product due to gene mutation, rather than the gene itself.

The current study found that heterozygous HTRA1 missense could lead to the arginine to glutamine change for the encoded protein located at 302nd amino acids. The mutation mode leads to a change in the structure of the protein, which may lead to changes in the spatial structure, and then to the change of the protein physical 
and chemical properties, physiological characteristics etc. Differently than HGMD, ESP6500, and dbSNP databases, this mutation has not been included in databases. Therefore, the SNP of HTRA1 is considered not present in the normal population, but present in the family members who are either diagnosed with cerebrovascular disease or positive in clinical imaging. The clinical imaging of subject III6 indicated the presence of disease but there was no clear clinical symptoms yet, which still needs further follow-ups. This result suggested that the genetic missense mutation was first observed in clinical imaging, and then in clinical manifestations. At the same time, we have found that onset of the disease occurred in younger family members, which complies with genetic characteristics of the early occurrence.

In summary, the heterozygous HTRA1 mutation is the cause of the disease, and the effects of the single gene

\section{References}

1. Pantoni L. Cerebral small vessel disease: from pathogenesis and clinical characteristics to therapeutic challenges. Lancet Neurol 2010; 9: 689-701, doi: 10.1016/S1474-4422 (10)70104-6.

2. Federico A, Di DI, Bianchi S, Di PC, Taglia I, Dotti MT. Hereditary cerebral small vessel diseases: a review. J Neurol Sci 2012; 322: 25-30, doi: 10.1016/j.jns.2012. 07.041.

3. Craggs LJL, Yamamoto Y, Deramecourt V, and Kalaria RN. Microvascular Pathology and Morphometrics of Sporadic and Hereditary Small Vessel Diseases of the Brain. Brain Pathol 2014; 24: 495-509, doi: 10.1111/bpa.12177.

4. Craggs LJ, Hagel C, Kuhlenbaeumer G, Borjessonhanson A, Andersen O, Viitanen $M$, et al. Quantitative vascular pathology and phenotyping familial and sporadic cerebral small vessel diseases. Brain Pathol 2013; 23: 547-557, doi: 10.1111/bpa.12041.

5. Wardlaw JM, Smith C, and Dichgans M. Mechanisms of sporadic cerebral small vessel disease: insights from neuroimaging. Lancet Neurol 2013; 12: 483-497, doi: 10.1016/S1474-4422(13)70060-7.

6. Joutel A, Corpechot C, Ducros A, Vahedi K, Chabriat H, Mouton $\mathrm{P}$, et al. Notch3 mutations in CADASIL, a hereditary adult-onset condition causing stroke and dementia. Nature 1996; 383: 707-710, doi: 10.1038/383707a0.

7. Service SK. Role of COL4A1 in Small-Vessel Disease and Hemorrhagic Stroke. N Engl J Med 2006; 354: 1489-1496, doi: $10.1056 /$ NEJMoa053727.

8. Zou Y, Zwolanek D, Izu Y, Gandhy S, Schreiber G, Brockmann $\mathrm{K}$, et al. Recessive and dominant mutations in COL12A1 cause a novel EDS/myopathy overlap syndrome in humans and mice. Hum Mol Genet 2014; 23: 2339-2350, doi: $10.1093 / \mathrm{hmg} / \mathrm{ddt} 627$.

9. Hara K, Shiga A, Fukutake T, Nozaki H, Miyashita A, Yokoseki $\mathrm{A}$, et al. Association of HTRA1 mutations and familial ischemic cerebral small-vessel disease. $N$ Engl $J$ Med 2017; 360: 1729-1739, doi: 10.1056/NEJMoa0801560.

10. Nishimoto $Y$, Shibata M, Nihonmatsu M, Nozaki H, Shiga A, Shirata A, et al. A novel mutation in the HTRA1 gene causes can be enhanced in the genetic process. However, further research is needed to understand the physicochemical properties and biological activity of the encoding protein caused by gene mutation. In addition, it is not clear whether the SNP is present in the normal population. Lastly, we selectively performed partial gene screening of the proband rather than the entire sequencing, so whether the gene mutation acts individually or in combination with other types of mutation for the family members is unknown and needs further investigation.

\section{Acknowledgments}

The authors thank Prof. YU Baofeng from the Department of Biochemistry at Shanxi Medical University for the help. This work was supported by the Applied and Basic Research Project in Shanxi Province (201701D121173).
CARASIL without alopecia. Neurology 2011; 76: $1353-$ 1355, doi: 10.1212/WNL.0b013e318215281d.

11. Wang XL, Li CF, Guo HW, and Cao BZ. A Novel Mutation in the HTRA1 Gene Identified in Chinese CARASIL Pedigree. CNS Neurosci Ther 2012; 18: 867-869, doi: 10.1111/j.17555949.2012.00373.x

12. Chen $\mathrm{Y}, \mathrm{He} Z$, Meng $\mathrm{S}$, Li L, Yang $\mathrm{H}$, and Zhang $\mathrm{X}$. A novel mutation of the high-temperature requirement $A$ serine peptidase 1 (HTRA1) gene in a Chinese family with cerebral autosomal recessive arteriopathy with subcortical infarcts and leukoencephalopathy (CARASIL). J Int Med Res 2013; 41: 1445-1455, doi: 10.1177/0300060513480926.

13. Bianchi S, Di PC, Gallus GN, Taglia I, Poggiani A, Rosini F, et al. Two novel HTRA1 mutations in a European CARASIL patient. Neurology 2014; 82: 898-900, doi: 10.1212/WNL. 0000000000000202.

14. Chai Z, Wang H, Changxin LI, and Xiaowei WU. Analysis of a pedigree with CADASIL-Like. Chin J Nerv Ment Dis 2013 $<$ http://xueshu.baidu.com/s?wd=paperuri\%3A\%287488814 4f770147481b15abae70c5027\%29\&filter=sc_long_sign\&tn= SE xueshusource $2 k d u w 22 v \& s c$ vurl=http $\% 3 A \% 2 F \% 2 F e n$. cnki.com.cn\%2FArticle_en\%2FCJFDTotal-ZSJJ201304001. htm\&ie $=u t f-8 \& s c$ us $=3529913461493540851>$.

15. Pantoni L, Pescini FS, Sarti C, Bianchi S, Dotti MT, Federico A, et al. Comparison of clinical, familial, and MRI features of CADASIL and NOTCH3-negative patients. Neurology 2010; 74: 57-63, doi: 10.1212/WNL.0b013e3181c7da7c.

16. Clausen $\mathrm{T}$, Southan $\mathrm{C}$, and Ehrmann $\mathrm{M}$. The HtrA family of proteases: implications for protein composition and cell fate. Mol Cell 2002; 10: 443-455, doi: 10.1016/S1097-2765(02)00658-5.

17. Nozaki $H$, Nishizawa $M$, and Onodera O. Features of cerebral autosomal recessive arteriopathy with subcortical infarcts and leukoencephalopathy. Stroke 2014; 45: 3447-3453, doi: 10.1161/STROKEAHA.114.004236.

18. Verdura E, Hervé D, Scharrer E, Amador MDM, Guyantmaréchal L, Philippi A, et al. Heterozygous HTRA1 mutations are associated with autosomal dominant cerebral small vessel disease. Brain 2015; 138: 2347-2358, doi: 10.1093/ brain/awv155. 